\title{
Cord Blood Mesenchymal Stromal Cell- Conditioned Medium Protects Endothelial Cells via STAT3 Signaling
}

\author{
Andreas Matthaeus Bader Andreja Brodarac $^{\mathrm{a}}$ Kristin Klose $^{\mathrm{a}}$ Karen Bieback $^{\mathrm{b}}$ \\ Yeong-Hoon Choic Kyung-Sun Kang ${ }^{d}$ Andreas Kurtz ${ }^{\mathrm{a}, \mathrm{d}}$ Christof Stamma,e
}

\begin{abstract}
aerlin-Brandenburg Center for Regenerative Therapies (BCRT), Berlin, Germany, 'bInstitute of Transfusion Medicine and Immunology, Mannheim, Germany, 'Heart Center, University of Cologne, Cologne, Germany, dCollege of Veterinary Medicine, Seoul National University, Seoul, Korea, e'Deutsches Herzzentrum Berlin, Berlin, Germany
\end{abstract}

\author{
Key Words \\ Cell therapy • Endothelial cell $\bullet$ Ischemia $\bullet$ Stem cell $\bullet$ Cord blood
}

\begin{abstract}
Background/Aims: Cell-based therapies may be useful for treating ischemic diseases, but the underlying mechanisms are incompletely understood. We investigated the impact of cord blood mesenchymal stromal cell (CBMSC)- or fibroblast (FB)-secreted factors on starved endothelial cells and determined the relevant intracellular signaling pathways. Methods: HUVECs were subjected to glucose/serum deprivation (GSD) in hypoxia or normoxia, in presence of CBMSC- or FB-conditioned medium (CM). Viability and proliferation were determined via WST-8 conversion and BrdU incorporation. Apoptosis was quantified by annexin V/ethidium homodimer-III staining, nuclear fragmentation and cell morphology. mRNA expression and protein phosphorylation were determined by real-time QPCR and western blot. Experiments were repeated in presence of small-molecule inhibitors. Results: The negative impact of GSD was most pronounced at $21 \% \mathrm{O}_{2}$. Here, medium of CBMSCs and FBs increased viability and proliferation and reduced apoptosis of HUVECs. This was associated with increased STAT3 and ERK1/2 phosphorylation and BCL-2 expression. Under STAT3 inhibition, the beneficial effect of CBMSC-CM on viability and BCL-2 expression was abolished. Conclusion: Factors released by CBMSCs protect endothelial cells from the deleterious impact of GSD by activation of the STAT3 survival pathway. However, this phenomenon is not CBMSC-specific and can be reproduced using juvenile fibroblasts.
\end{abstract}




\section{Introduction}

Mesenchymal stromal cells (MSC) and their subpopulations have been shown to ameliorate ischemic tissue injury in numerous pre-clinical studies. Clinical trials using MSCs for treatment of ischemic diseases gave promising results but also demonstrated the need for further improvement [1-3]. The beneficial MSC effects range from improved vascularization and perfusion to the protection of ischemically damaged cells and favorable influences on tissue remodeling $[4,5]$, notably in cardiac and skeletal muscle [6-8]. The impact on capillary density and collateral flow has been attributed to secreted factors $[8,9]$, but little is known about how these factors act on vascular cells in the ischemic area. We therefore established an in vitro model of "simulated ischemia" using human umbilical vein endothelial cells (HUVEC) and studied whether factors released from MSCs provide protective stimuli. We used MSCs from umbilical cord blood (CBMSC) because they are a uniform cell population with low immunogenicity, ready availability and robust culture and expansion behavior, and clinical trials have demonstrated their therapeutic potential for the treatment of ischemic diseases $[1,10]$. To assess the MSC-specificity of these effects, we also investigated the response of endothelial cells to medium conditioned by juvenile fibroblasts (FB). We believe that a better understanding of the underlying molecular mechanisms may ultimately facilitate their more successful clinical application.

\section{Materials and Methods}

\section{Cells and cell culture}

Cryopreserved HUVECs were purchased from Life Technologies, Carlsbad, California, USA. Cells were maintained in endothelial basal medium (EBM)-2 supplemented with endothelial growth medium (EGM)2 growth factors, cytokines and supplements (Lonza, Basel, Switzerland) with $10 \%$ fetal bovine serum (FBS) (Life Technologies, Carlsbad, California, USA), and cultured in $0.1 \%$ gelatin coated flasks and plates (Thermo Fisher Scientific, Waltham, Massachusetts, USA) at $37{ }^{\circ} \mathrm{C}$ and $5 \% \mathrm{CO}_{2}$ in a humid atmosphere. All experiments were performed with HUVECs between passage seven and nine, $24 \mathrm{~h}$ after plating.

Cryopreserved human CBMSCs were provided by Karen Bieback, who isolated, expanded and characterized them according to a previously published protocol [11]. Cord blood was obtained with informed consent of the mother, according to the principles outlined in the Declaration of Helsinki and with approval of the local ethical committees in Mannheim and Heidelberg (Ref. 48/05 reconfirmed in 2009). With a seeding density of $700-1000$ cells $/ \mathrm{cm}^{2}$ and harvesting at subconfluency, CBMSCs were expanded to the fourth passage and cultured in Dulbecco's Modified Eagle Medium (DMEM) 21885 (low glucose), supplemented with $10 \% \mathrm{FBS}, 100 \mathrm{U} / \mathrm{ml}$ penicillin, and $100 \mu \mathrm{g} / \mathrm{ml}$ streptomycin at $37{ }^{\circ} \mathrm{C}$ and $5 \% \mathrm{CO}_{2}$ in a humid atmosphere (all reagents from Life Technologies, Carlsbad, California, USA). The phenotype of the cultured MSCs as well as their ability to differentiate into non-hematopoietic cell types were previously confirmed $[12,13]$. CRL-2429 ${ }^{\mathrm{Tm}}$ fibroblasts from human foreskin were purchased in passage four from LGC Standards, Wesel, Germany. With a seeding density of 10000 cells $/ \mathrm{cm}^{2}$ and harvesting at 95-100\% confluency, cells were cultured in Iscove's Modified Dulbecco's Medium (IMDM) 31980, supplemented with $10 \% \mathrm{FBS}, 100 \mathrm{U} / \mathrm{ml}$ penicillin, and $100 \mu \mathrm{g} / \mathrm{ml}$ streptomycin at $37{ }^{\circ} \mathrm{C}$ and $5 \% \mathrm{CO}_{2}$ in a humid atmosphere (all reagents from Life Technologies, Carlsbad, California, USA).

\section{Preparation of conditioned medium}

Conditioned medium was prepared using CBMSCs in passage four and FBs between passages ten and twelve. Cells were plated at 10000 cells $/ \mathrm{cm}^{2}$. After $6 \pm 1$ (CBMSC) or $3 \pm 1$ (FB) days, at $80-90 \%$ confluence, cells were washed twice with Dulbecco's Phosphate Buffered Saline (DPBS) and covered with DMEM 11966 (no glucose), supplemented with $100 \mathrm{U} / \mathrm{ml}$ penicillin and $100 \mu \mathrm{g} / \mathrm{ml}$ streptomycin (all reagents from Life Technologies, Carlsbad, California, USA). Subsequently, cells were subjected to a hypoxic atmosphere $\left(1 \% \mathrm{O}_{2}\right.$, $5 \% \mathrm{CO}_{2}$ ) by replacing oxygen with nitrogen in an $\mathrm{O}_{2}$ and $\mathrm{CO}_{2}$ controlled multi gas incubator (CB 150, Binder, Tuttlingen, Germany). After six days' incubation, conditioned medium was collected and centrifuged for 10 min at $300 \mathrm{xg}$ to remove detached cells. Equivalent medium kept under the same conditions in culture flasks 
Bader et al.: Endothelial Protection by MSC

without cells was prepared as control. For depletion of extracellular microvesicles, conditioned medium was filtered through a $0.2-\mu \mathrm{m}$ filter and subsequently centrifuged for $90 \mathrm{~min}$ at $100000 \mathrm{x} \mathrm{g}$, according to a published protocol [14]. Concentration of the medium by volume reduction was not performed. CBMSC/ fibroblast viability after conditioned medium preparation was verified as previously described [12].

"Simulated ischemia"

HUVECs were subjected to combined glucose/serum deprivation (GSD) by replacing their standard culture medium with CBMSC- or FB-conditioned medium or with equivalent non-conditioned control medium. In these media HUVECs were incubated under normoxic conditions $\left(21 \% \mathrm{O}_{2}, 5 \% \mathrm{CO}_{2}\right)$ or subjected to a hypoxic environment $\left(0.5 \% \mathrm{O}_{2}, 5 \% \mathrm{CO}_{2}\right)$ in an incubator chamber (Billups-Rothenberg, Del Mar, California, USA and BioSpherix, Lacona, New York, USA). HUVECs incubated under normoxic conditions in DMEM 31966 (high glucose) supplemented with $10 \%$ FBS, $100 \mathrm{U} / \mathrm{ml}$ penicillin, and $100 \mu \mathrm{g} / \mathrm{ml}$ streptomycin served as reference (= full medium) (all reagents from Life Technologies, Carlsbad, California, USA).

Evaluation of cell damage

Metabolic activity was determined using the Cell Counting Kit-8 (Dojindo, Kumamoto, Japan). After $6 \mathrm{~h}$ of GSD, HUVECs were incubated for $4 \mathrm{~h}$ in medium containing water soluble tetrazolium (WST)-8. Absorbance at $450 \mathrm{~nm}$, resulting from the conversion of WST-8 to WST-8 formazan by metabolically active cells, and at $650 \mathrm{~nm}$ (reference) was measured using a standard micro plate reader. The number of HUVECs showing morphological signs of apoptosis was determined by manual observation. After $6 \mathrm{~h}$ of simulated ischemia, light microscopy images were taken of the center of each microplate well. Only cells exhibiting a typical apoptotic morphology (shrinkage, rounding, and/or membrane blebbing) were counted. To further characterize the mode of cell death, the Apoptotic/Necrotic Cells Detection Kit (PromoKine, Heidelberg, Germany) was used. After $6 \mathrm{~h}$ GSD in 96-well imaging plates (PerkinElmer, Rodgau-Jügesheim, Germany), cells were stained with annexin $\mathrm{V}$ (AnnV)-fluorescein isothiocyanate (FITC) and ethidium homodimer (EthD)-III, fixed with 4\% paraformaldehyde (PFA) and nuclei were counterstained with 4',6-diamidino2-phenylindole (DAPI). The total cell number and proportions of early apoptotic, late apoptotic and necrotic cells were quantified using the high content imaging system Operetta $₫$ and Harmony ${ }^{\circledR}$ software (PerkinElmer, Waltham, MA, USA). In separate experiments, nuclear fragmentation was quantified: cells were fixed with $4 \%$ PFA, nuclei were stained with DAPI and analyzed with the Operetta ${ }^{\circledR}$ system. The nuclear fragmentation index (NFI) was defined as the coefficient of variation of nuclear stain fluorescence intensity. 5-bromo-2-deoxyuridine (BrdU) uptake was determined with the Cell Proliferation ELISA, BrdU (Roche Diagnostics, Mannheim, Germany). Cells were incubated with BrdU during the final $3 \mathrm{~h}$ of the GSD period, and cells were processed according to the manufacturer's instructions. Absorbance at $370 \mathrm{~nm}$ and $492 \mathrm{~nm}$ (reference) was measured using a standard microplate reader.

\section{Gel electrophoresis and western blot analysis}

Cells were washed with DPBS and lysed in PRO-PREPTM Protein Extraction Solution (Intron Biotechnology, Sungnam, South Korea). Protein concentration was determined by $\mathrm{DC}^{\mathrm{TM}}$ Protein Assay (Bio-Rad, Hercules, California, USA) and denatured protein ( $30 \mu \mathrm{g}$ ) was resolved in a $12 \%$ polyacrylamide sodium dodecyl sulfate (SDS) gel and transferred to nitrocellulose membranes, which were blocked and incubated with monoclonal mouse anti-total-protein and rabbit anti-phospho-protein primary antibodies overnight at $4{ }^{\circ} \mathrm{C}$ : Akt, phospho-Akt (Ser473), extracellular-signal-regulated kinase (ERK)1/2, phosphoERK1/2 (Thr202/Tyr204), signal transducer and activator of transcription (STAT)3, phospho-STAT3 (Tyr705) (all from Cell Signaling Technology, Danvers, Massachusetts, USA) and glyceraldehyde 3-phosphate dehydrogenase (GAPDH) (Merck Millipore, Darmstadt, Germany). After $1 \mathrm{~h}$ incubation with goat anti-mouse IgG $(\mathrm{H}+\mathrm{L})$ / goat anti-rabbit IgG $(\mathrm{H}+\mathrm{L})$ horseradish peroxidase conjugated secondary antibodies (Life Technologies, Carlsbad, California, USA), blots were analyzed using Amersham ${ }^{\text {TM }}$ ECL $^{\text {TM }}$ Western blotting detection reagents (GE Healthcare, Little Chalfont, UK).

Real time quantitative PCR (RT-qPCR)

Cells were washed with DPBS and RNA was purified using the RNeasy® Mini Kit (Qiagen, Hilden, Germany). cDNA was synthesized from DNase (Sigma-Aldrich, St. Louis, Missouri, USA) -treated total RNA using the SuperScript ${ }^{\circledR}$ III First-Strand Synthesis System for RT-PCR (Life Technologies, Carlsbad, 
California, USA) with random hexamers as reaction primers. RT-qPCR was performed in a Mastercycler ${ }^{\circledR}$ ep gradient $\mathrm{S}$ realplex ${ }^{2}$ (Eppendorf, Hamburg, Germany), using $2.5 \mathrm{ng}$ template in $25 \mu \mathrm{l}$ reaction volume with $300 \mathrm{nM}$ of each primer and 2 x Power SYBR ${ }^{8}$ Green PCR Master Mix (Life Technologies, Carlsbad, California, USA). Amplification conditions were as follows: $95^{\circ} \mathrm{C}$ for $10 \mathrm{~min}$ followed by 35 cycles consisting of $95^{\circ} \mathrm{C}$ for $15 \mathrm{~s}, 56{ }^{\circ} \mathrm{C}$ for $30 \mathrm{~s}, 68^{\circ} \mathrm{C}$ for $60 \mathrm{~s}$. For B-cell lymphoma-2 (BCL-2) only $150 \mathrm{nM}$ primers were used and annealing temperature was set to $60^{\circ} \mathrm{C}$. The following oligonucleotides were used as forward and reverse primers respectively: 5'-CATGTACGTTGCTATCCAGGC-3' and 5'- CTCCTTAATGTCACGCACGAT-3' for beta-actin, 5'-TGAGAAGCACGACCTTCATGT-3' and 5'-GGAACCCCTATGACCTCTTCA-3' for BCL2-associated athanogene (BAG-1), 5'- GAACTGGGGGAGGATTGTGG-3' and 5'- GCCGGTTCAGGTACTCAGTC-3' for BCL-2, 5'-GAGCTGGTGGTTGACTTTCTC-3' and 5'-TCCATCTCCGATTCAGTCCCT-3' for B-cell lymphoma-extra-large (BCL-XL). All measurements were carried out in triplicates. Gene-of-interest expression (E) was calculated as $\mathrm{E}=$ primer efficiency ${ }^{(-\mathrm{Ct})}$ (where $\mathrm{C}_{\mathrm{t}}$ is the number of cycles at which the fluorescence exceeds the threshold) and normalized to beta-actin expression.

Signaling pathway inhibition

HUVECs were treated with specific small-molecule inhibitors $1 \mathrm{~h}$ prior to and during simulated ischemia. The MEK1/2 inhibitor U0126 (Cell Signaling Technology, Danvers, Massachusetts, USA) and the STAT3 inhibitor Stattic (Merck Millipore, Darmstadt, Germany) were applied at a final concentration of 5 $\mu \mathrm{M}$ and $7.5 \mu \mathrm{M}$, respectively. Both inhibitors were diluted in dimethyl sulfoxide (DMSO) and an equivalent amount of solvent was used for control.

\section{Statistical analysis}

Results are expressed as means \pm SEM. All cell culture experiments and PCR analyses were performed in triplicates and the sample size (n) indicates the number of triplicates. The significance of intergroup differences was determined by one-way analysis of variance (ANOVA) with two-tailed Dunnett's t-test. When only two groups were compared, a two-tailed Student's t-test was performed. IBM SPSS statistics 20 was used for data analysis. A P-value $<0.05$ was considered significant.

\section{Results}

Validation of the "simulated ischemia" model in HUVECS

In order to mimic ischemic conditions in vitro, HUVECs were deprived of glucose and serum while kept in a hypoxic atmosphere $\left(0.5 \% \mathrm{O}_{2}\right)$, and the effect of GSD was also investigated in normoxia $\left(21 \% \mathrm{O}_{2}\right)$. Metabolic activity, total cell number, apoptosis and proliferation rate were compared to those of HUVECs supplied with full medium. After $6 \mathrm{~h}$ of GSD, WST-8 conversion of the cells declined to $87 \pm 5 \%$ in hypoxia and further to $69 \pm 3 \%$ in normoxia ( $\mathrm{P}<0.01$ vs. hypoxia) (Fig. 1A). Accordingly, the proportion of remaining adherent cells was $80 \pm 3 \%$ in hypoxia but only $59 \pm 3 \%$ in normoxia ( $P<0.001$ vs. hypoxia) (Fig. 1B). BrdU uptake decreased to $0.7 \pm 0.05$ (normalized to full medium data) in hypoxia, but even further to $0.42 \pm 0.08$ in normoxia ( $P<0.01$ vs. hypoxia) (Fig. 1C). The number of cells showing an apoptotic phenotype was enhanced by factor $8 \pm 1$ in hypoxia and by factor $10 \pm 1$ in normoxia (Fig. 1D). In summary, withdrawal of glucose and serum for 6 hours impaired the viability and proliferation of HUVECs, but the deleterious effect was more pronounced in presence of $21 \%$ oxygen. Leaving sufficient room for both, improvement or further deterioration, normoxic GSD appeared to be more suitable and was therefore used for our conditioned medium experiments.

Protective effects of CBMSC- and FB-conditioned medium on glucose/serum deprived HUVECS

WST-8 conversion was higher in the presence of either CBMSC- or FB-conditioned medium (CBMSC-CM: $89 \pm 7 \%$ of full medium and FB-CM: $96 \pm 5 \%$; vs. control: $69 \pm 3 \%, \mathrm{P}<$ 0.05) (Fig. 2A) and the number of remaining adherent cells was correspondingly higher (CBMSC-CM: $93 \pm 2 \%$ of full medium and FB-CM: $91 \pm 2 \%$; vs. control: $59 \pm 3 \%, \mathrm{P}<0.001$ ) 


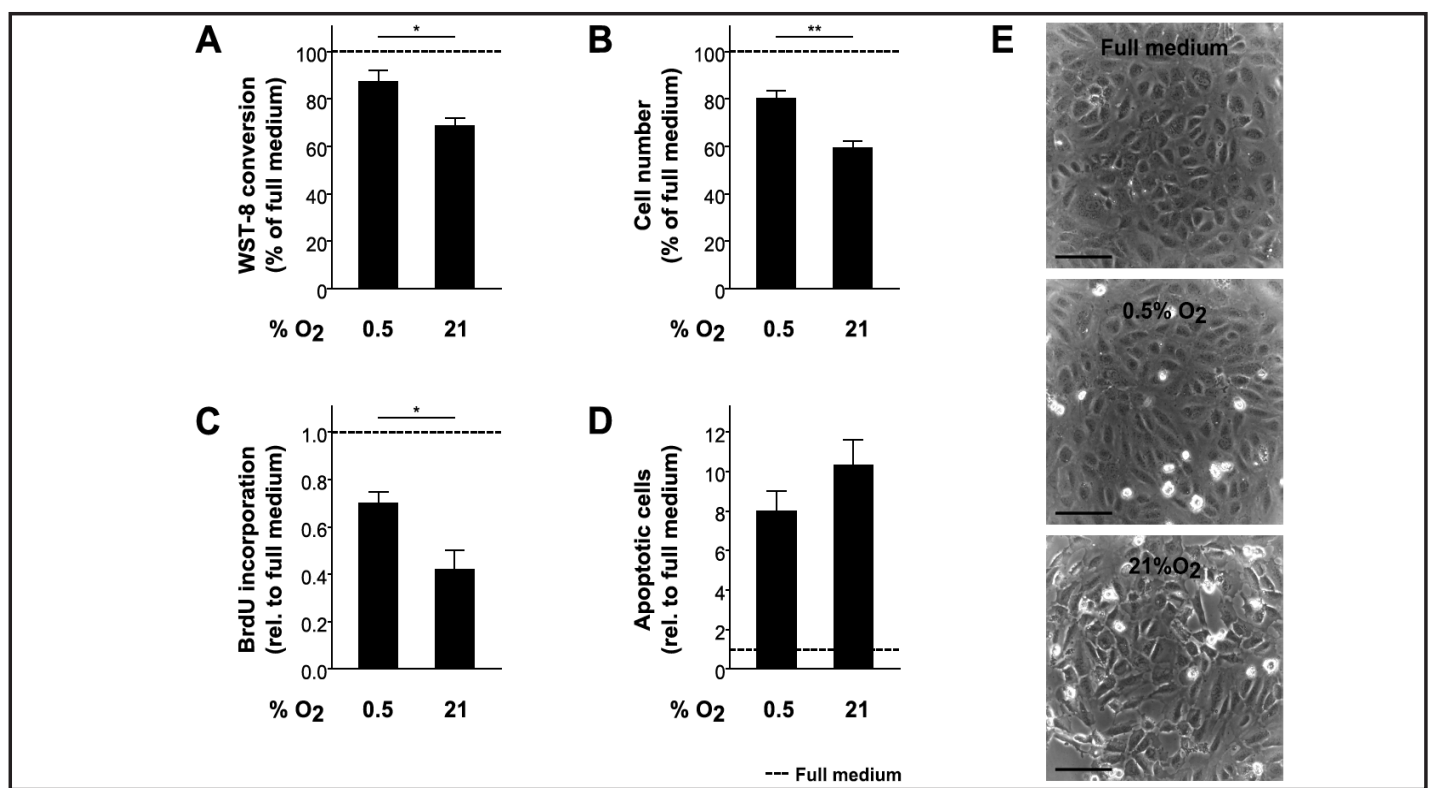

Fig. 1. Viability, proliferation and apoptosis of glucose/serum deprived HUVECs. (A) WST-8 conversion $(0.5 \%: n=8 ; 21 \%: n=8)$, (B) cell number $(0.5 \%: n=6 ; 21 \%: n=13)(C)$ BrdU incorporation $(0.5 \%: n=12$; $21 \%: n=13)$ and (D) frequency of cells exhibiting morphological characteristics of apoptosis $(0.5 \%: n=7$; 21\%: $\mathrm{n}=11$ ) after $6 \mathrm{~h}$ glucose/serum deprivation at $0.5 \% \mathrm{O}_{2}$ and $21 \% \mathrm{O}_{2}\left({ }^{*} \mathrm{P}<0.01,{ }^{* *} \mathrm{P}<0.001\right.$; Student's t-test). All values expressed in relation to glucose/serum supplied cells cultured at $21 \% \mathrm{O}_{2}$ (Full medium). Data shown as mean \pm SEM. (E) Representative microphotographs taken by light microscopy, scale bar = $100 \mu \mathrm{m}$.

(Fig. 2B). Also BrdU incorporation was preserved in conditioned medium (CBMSC-CM: $0.92 \pm 0.03$-fold of full medium and FB-CM: 0.95 \pm 0.02 -fold; vs. control $0.42 \pm 0.08$-fold, $\mathrm{P}<$ 0.001 ) (Fig. 2C). The frequency of cells showing morphological characteristics of apoptosis was reduced in conditioned medium (CBMSC-CM: $5 \pm 1$-fold increase compared to full medium and FB-CM: $6 \pm 1$-fold increase; vs. control: 10 \pm 1 -fold increase, $\mathrm{P}<0.05$ ) (Fig. 2D), and the nuclear fragmentation index was lower (CBMSC-CM: NFI $0.151 \pm 0.003$ and FBCM: NFI $0.150 \pm 0.004$; vs. control: NFI $0.175 \pm 0.002, \mathrm{P}<0.001$ ) (Fig. 2E). The rate of cells exposing phosphatidylserine with preserved plasma membrane integrity (early apoptosis) was also reduced in conditioned medium (CBMSC-CM: $0.58 \pm 0.06$ of control, $\mathrm{P}<0.001$; FB$\mathrm{CM}$ : $0.66 \pm 0.07$ of control, $\mathrm{P}<0.01$ ), as was the rate of non-phosphatidylserine-exposing but EthD-III permeable cells (necrosis) (CBMSC-CM: $0.47 \pm 0.06$ of control, $\mathrm{P}<0.01$; FB-CM: $0.33 \pm 0.03$ of control, $\mathrm{P}<0.001$ ) (Fig. 3).

\section{Effect of soluble factors and extracellular microvesicles in CBMSC-conditioned medium}

As we have previously shown, both CBMSC- and FB-conditioned medium contain growth factors like vascular endothelial growth factor (VEGF), epidermal growth factor (EGF), hepatocyte growth factor (HGF) and basic fibroblast growth factor (bFGF) [12]. In addition to such soluble factors, protein- and RNA-containing microvesicles are also known to be released from cells in the culture medium [14]. To test whether the beneficial effects of conditioned medium were mediated by extracellular vesicles, we repeated the GSD experiment with microvesicle-depleted CBMSC-conditioned medium. Neither $0.2-\mu \mathrm{m}$ filtration nor subsequent ultracentrifugation at $100000 \mathrm{x}$ g lowered the effect of CBMSCconditioned medium on metabolic activity of glucose/serum deprived HUVECs (Fig. 4).

Signaling pathway regulation in glucose/serum deprived HUVECS

Compared to cells incubated in full medium, GSD resulted in similar phosphorylation of Akt and STAT3, while ERK1/2 phosphorylation was 2.5 \pm 0.4 -fold increased (Fig. 5A). 


\section{A}

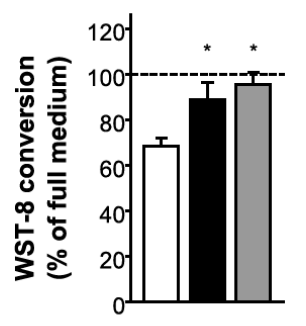

D

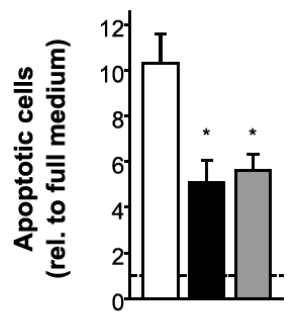

$\mathbf{F}$

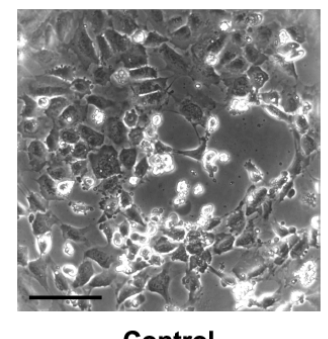

B

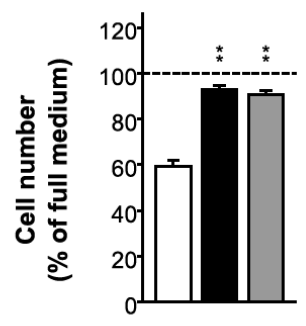

$\mathbf{E}$
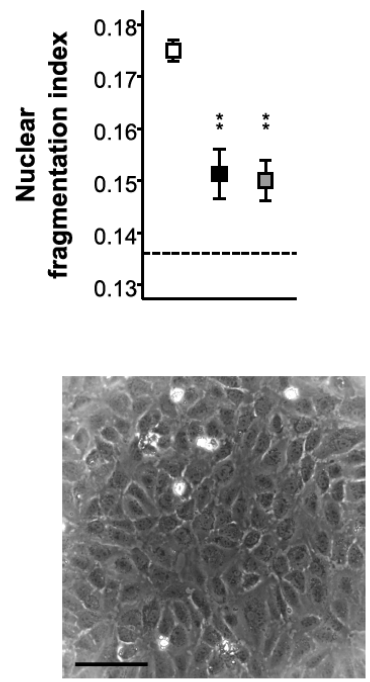

CBMSC-CM
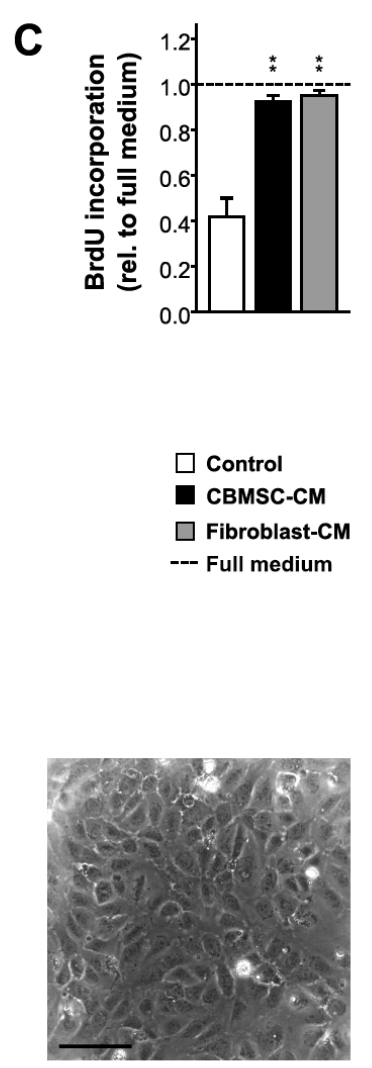

Fibroblast-CM

Fig. 2. Survival, proliferation and apoptosis of HUVECs after glucose/serum deprivation in CBMSC- and FBconditioned medium. (A) WST-8 conversion (control: $n=8$; CBMSC-CM: $n=5$; FB-CM: $n=5$ ), (B) cell number (control: $\mathrm{n}=13$; CBMSC-CM: $\mathrm{n}=8$; FB-CM: $\mathrm{n}=8$ ), (C) BrdU incorporation (control: $\mathrm{n}=13$; CBMSC-CM: $\mathrm{n}=$ 8; FB-CM: $n=5$ ), (D) frequency of cells exhibiting morphological characteristics of apoptosis (control: $n=$ 11; CBMSC-CM: $n=7$; FB-CM: $n=8$ ) and (E) nuclear fragmentation (control: $n=21$; CBMSC-CM: $n=12$; FB$\mathrm{CM}: \mathrm{n}=12$ ) after $6 \mathrm{~h}$ normoxic glucose/serum deprivation $\left(* \mathrm{P}<0.05\right.$, ${ }^{* *} \mathrm{P}<0.001$ vs. control; ANOVA with Dunnett's t-test). (A-D) Values expressed in relation to glucose/serum supplied cells (Full medium). Data shown as mean \pm SEM. (F) Representative microphotographs taken by light microscopy, scale bar $=100 \mu \mathrm{m}$.

In the presence of conditioned medium, however, we detected distinct differences in the phosphorylation pattern of all three survival pathway checkpoints. Phosphorylation of Akt decreased to $0.6 \pm 0.1$ in CBMSC-CM ( $\mathrm{P}<0.05$ vs. control), and we observed a trend toward a similar reduction in FB-CM $(0.6 \pm 0.2, \mathrm{P}=0.05$ vs. control). Phosphorylation of STAT3 increased $10 \pm 1$-fold in CBMSC-CM ( $P<0.001$ vs. control) and 5.6 \pm 0.5 -fold in FB-CM $(\mathrm{P}<0.01$ vs. control). ERK1/2-phosphorylation was $6 \pm 2$-fold higher in FB-CM (P $<0.05$ vs. control), while in CBMSC-CM, the average ERK1/2-phosphorylation was $4.5 \pm 0.8$-fold higher but did not reach statistical significance ( $\mathrm{P}=0.3$ vs. control) (Fig. $5 \mathrm{~A})$.

Modulation of gene expression in glucose/serum deprived HUVECS

BCL-2 mRNA expression was similar in glucose/serum-deprived cells and cell incubated in full medium, while mRNA expression of BCL-XL and BAG-1 was 1.6 \pm 0.1 -fold and 1.6 \pm 0.2 fold higher in GSD (Fig. 5C). In the presence of CBMSC-CM, BCL-2 expression increased $3.0 \pm 0.3$-fold ( $\mathrm{P}<0.001$ vs. control) and $2.0 \pm 0.2$-fold in FB-CM ( $\mathrm{P}<0.01$ vs. control). Neither BCL-XL nor BAG-1 was further induced in the presence of conditioned medium (Fig. 5C). 


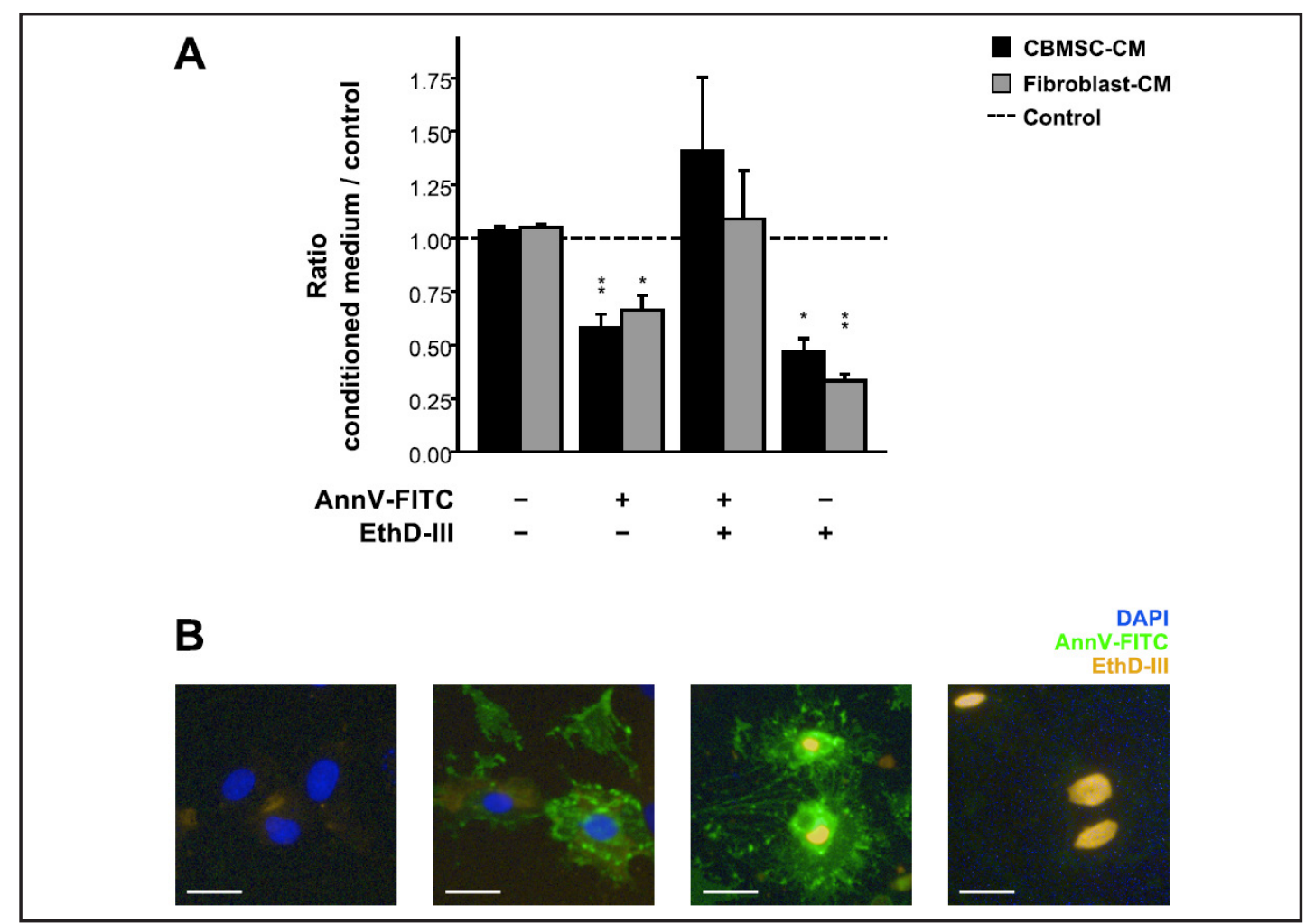

Fig. 3. Phosphatidylserine exposure and loss of plasma membrane integrity of HUVECs after glucose/ serum deprivation in CBMSC- and FB-conditioned medium. (A) Percentage of AnnV-FITC binding and EthDIII permeable cells after $6 \mathrm{~h}$ normoxic glucose/serum deprivation in CBMSC-CM ( $\mathrm{n}=8)$ and FB-CM $(n=8)$, expressed in relation to non-conditioned control medium $\left(* \mathrm{P}<0.01,{ }^{* *} \mathrm{P}<0.001\right.$ vs. control; ANOVA with Dunnett's t-test). Data shown as mean \pm SEM. (B) Representative microphotographs of vital (AnnV-FITC -/ EthD-III -), early apoptotic (AnnV-FITC + /EthD-III -), late apoptotic (AnnV-FITC +/EthD-III +) and necrotic $($ AnnV-FITC -/EthD-III +) HUVECs taken by Operetta® high-content imaging microscopy, scale bar $=25 \mu \mathrm{m}$.

Fig. 4. Effect of microvesicle depleted CBMSCconditioned medium. Viability (WST-8 conversion) of HUVECs after $6 \mathrm{~h}$ normoxic glucose/serum deprivation in untreated $(\mathrm{n}=6), 0.2-\mu \mathrm{m}$ filtered $(\mathrm{n}$ = 6) and $100000 \times$ g centrifuged $(n=6)$ CBMSC-CM, expressed in relation to non-conditioned control medium ( $* \mathrm{P}<0.001$ vs. control; ANOVA with Dunnett's t-test). Data shown as mean \pm SEM.

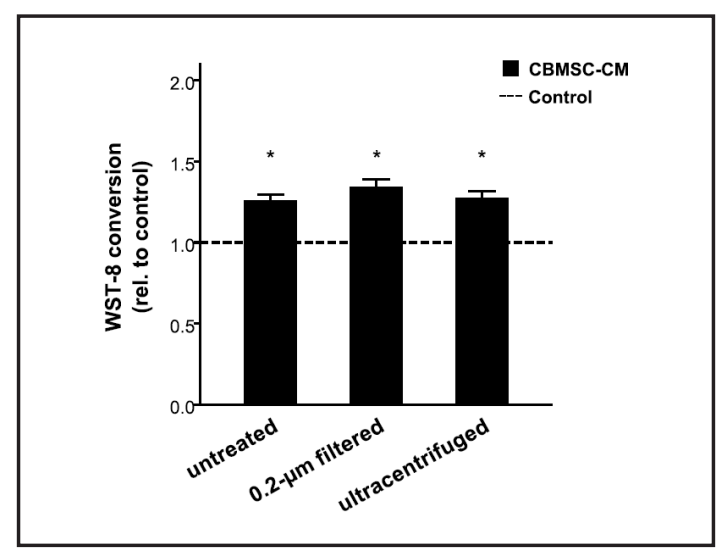

Functional relevance of CBMSC-conditioned medium triggered STAT3 activation

GSD experiments with CBMSC-conditioned medium were repeated in the presence of specific small-molecule inhibitors. The efficiency of Stattic and U0126 in suppressing the phosphorylation of STAT3 and ERK1/2 in glucose/serum deprived HUVECs was confirmed by western blot (Fig. 6A). The WST-8 conversion rate of glucose/serum starved HUVECs was increased in CBMSC-CM $(1.34 \pm 0.04$-fold of control, $\mathrm{P}<0.01)$. In the presence of the STAT3 inhibitor Stattic, this beneficial effect was completely abolished (1.0 \pm 0.2 -fold of control, $\mathrm{P}=1$ ), whereas blocking MEK/ERK signaling with U0126 did not influence the CBMSC-CM 

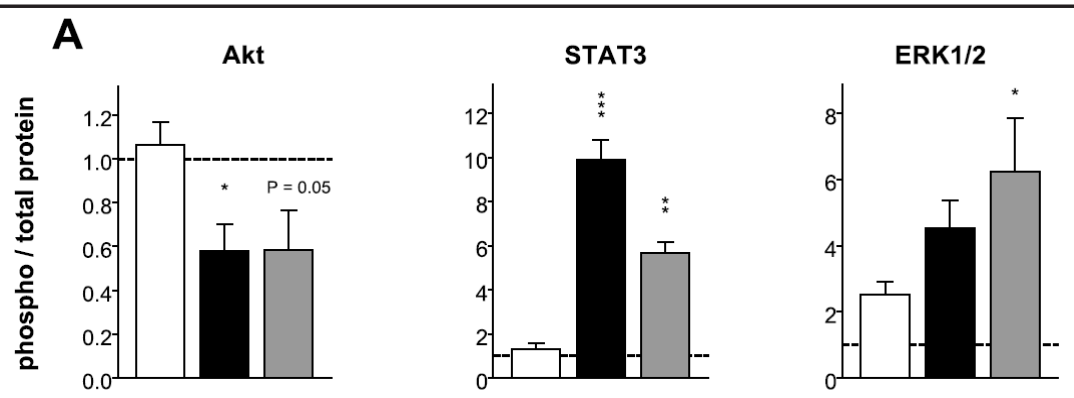

B
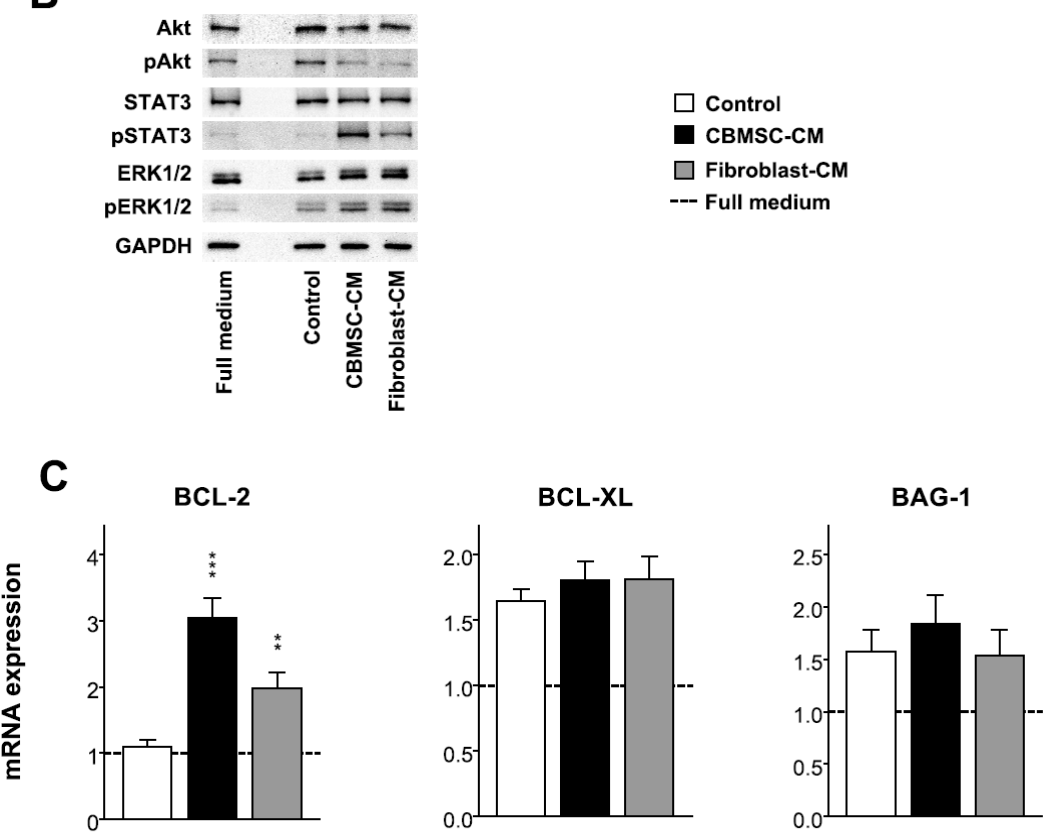

Fig. 5. Protein phosphorylation and gene expression in HUVECs after glucose/serum deprivation in CBMSCand FB-conditioned medium. (A) Phosphorylation of Akt (control: $n=6$; CBMSC-CM: $n=6$; FB-CM: $n=4$ ), STAT3 (control: $\mathrm{n}=6$; CBMSC-CM: $\mathrm{n}=6$; FB-CM: $\mathrm{n}=4$ ) and ERK1/2 (control: $\mathrm{n}=5$; CBMSC-CM: $\mathrm{n}=5$; FBCM: $n=4$ ) after 3 h normoxic glucose/serum deprivation, determined by western blot. (B) Representative western blot images. (C) beta-actin normalized mRNA expression of BCL-2 (control: $n=11$; CBMSC-CM: $n$ = 7; FB-CM: $n=7$ ), BCL-XL (control: $\mathrm{n}=11$; CBMSC-CM: $\mathrm{n}=7$; FB-CM: $\mathrm{n}=7$ ) and BAG-1 (control: $\mathrm{n}=11$; CBMSC-CM: $\mathrm{n}=7$; FB-CM: $\mathrm{n}=7$ ) after 3 h normoxic glucose/serum deprivation, determined by RT-qPCR (* $\mathrm{P}<0.05$; ${ }^{* *} \mathrm{P}<0.01,{ }^{* * *} \mathrm{P}<0.001$ vs. control; ANOVA with Dunnett's t-test). All values expressed in relation to glucose/serum supplied cells. Data shown as mean \pm SEM.

effect (1.57 \pm 0.06 -fold of control, $\mathrm{P}<0.001$ ) (Fig. 6B). Of note, CBMSC-CM triggered induction of BCL-2 $(2.4 \pm 0.1$-fold of control, $\mathrm{P}<0.001)$ was completely eliminated under STAT3 inhibition (1.0 \pm 0.2 -fold of control, $\mathrm{P}=1$ ) (Fig. 6C).

\section{Discussion}

We found that the deleterious impact of glucose/serum deprivation on HUVECs is more pronounced in normoxia than in hypoxia. Soluble factors secreted by umbilical cord blood MSCs act anti-apoptotic and pro-mitotic on the starved endothelial cells, but factors released 


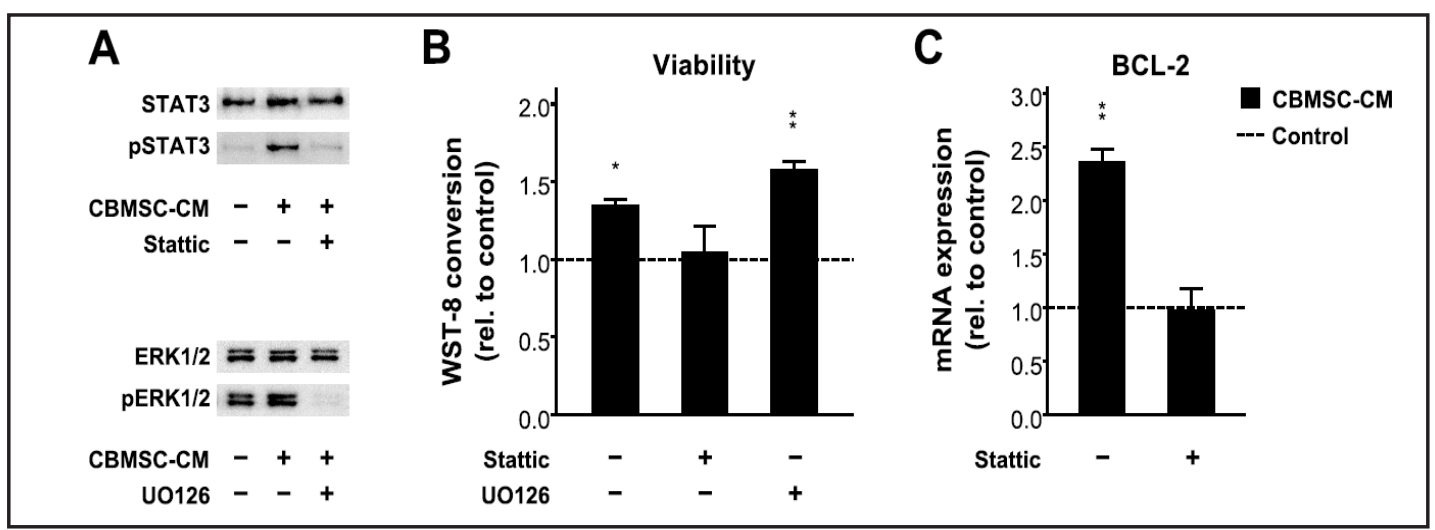

Fig. 6. Functional relevance of CBMSC-conditioned medium triggered STAT3 activation. (A) Protein phosphorylation and its inhibition by small-molecule inhibitors determined by western blot. (B) Viability determined via WST-8 conversion assay (solvent: $n=15$; Stattic: $n=8$; U0126: $n=15$ ). (C) beta-actin normalized mRNA expression of BCL-2, determined via RT-qPCR (solvent: $n=6$; Stattic: $n=6)(* P<0.01$, ** $\mathrm{P}<0.001$ vs. control; ANOVA with Dunnett's t-test). Data collected on HUVECs after (A and C) $3 \mathrm{~h}$ or (B) $6 \mathrm{~h}$ normoxic glucose/serum deprivation in CBMSC-CM, CBMSC-CM with 7.5 $\mu$ M Stattic and CBMSC-CM with 5 $\mu \mathrm{M}$ U0126. All values expressed in relation to non-conditioned control medium. Data shown as mean \pm SEM.

by fibroblasts exert similar effects. STAT3, but not ERK1/2 transmits the protective signals in endothelial cells and is needed for the induction of the anti-apoptotic BCL-2 gene.

To develop a suitable in vitro model for ischemia, we subjected HUVECs to combined glucose/serum deprivation in both hypoxia and normoxia. Under hypoxic conditions survival and proliferation of the cells were impaired, while in a normoxic atmosphere cell damage was even more severe. For glucose/serum starved HUVECs, hypoxia apparently acts as protective factor, while other groups described deleterious effects of hypoxia on HUVECs and mature endothelial cells kept in normal medium [15-17]. The damage to glucose/ serum starved HUVECs was alleviated by CBMSC-conditioned medium, which reduced apoptotic and necrotic cell death and increased the number of metabolically active cells with preserved proliferative capacity. Recent reports described favorable effects of MSC-derived microvesicles on endothelial cells $[18,19]$, but we found that the beneficial action on starved HUVECs does not depend on such structures. Anti-apoptotic and pro-mitotic effects of MSCs on endothelial cells have been described before $[18,20,21]$, but the underlying mechanisms have rarely been investigated. The phosphatidylinositol-4,5-bisphosphate 3-kinase (PI3K)/ Akt and mitogen-activated protein kinase (MEK)/ERK pathways typically transduce signals promoting survival and proliferation [22, 23]. Hung et al. reported that activation of Akt, but not ERK is responsible for beneficial effects of bone marrow MSC-conditioned medium on oxygen/serum-deprived endothelial cells [24]. Our findings confirm that MSC-triggered ERK activation is not required for protection, but CBMSC-released factors clearly suppressed Akt phosphorylation in our model. The janus kinase (JAK)/STAT pathway is also known to mediate cytoprotection $[25,26]$. As we and others have shown before, MSC-secreted factors are able to activate STAT3 in skeletal and cardiac myocytes as well as in cardiac progenitor cells [12, 27-29]. However, STAT3 is not in every cell type a key mediator of the cytoprotective stem cell action [12]. In our model, STAT3 was prominently activated in HUVECs in the presence of CBMSC-conditioned medium, and when STAT3 phosphorylation was blocked, the beneficial effect was completely abolished. Induction of anti-apoptotic genes BCL2 , BCL-XL or BAG-1 is one mechanism by which these pathways can promote survival of injured cells [30-32]. While BCL-XL and BAG-1 were up-regulated in HUVECs as endogenous response to glucose/serum deprivation, BCL-2 expression was triggered by CBMSC-released factors in the medium. Confirming the crucial role of STAT3, BCL-2 induction was completely abolished when STAT3 phosphorylation was blocked. Such anti-apoptotic action of STAT3mediated BCL-2 induction has also been described in polyamine-depleted cells [30]. 
To determine whether non-stem cells exert similar beneficial effects, we included juvenile foreskin fibroblasts in our experiments. As we have shown before, both cell types differ in their secretory profile [12]. While secreting comparable amounts of VEGF, EGF and Angiopoetin-2, fibroblasts release less HGF but higher amounts of bFGF and interleukin 6 (IL-6) [12]. Yet, we found that their anti-apoptotic and pro-mitotic action on endothelial cells was similar to that of CBMSCs. Although STAT3 phosphorylation and subsequent BCL2 expression were less pronounced, activation of the STAT3/BCL-2 axis was sufficient to convey cytoprotection. Increased DNA synthesis and BCL-2 expression has previously been observed in oxygen/serum-deprived HUVECs in the presence of cardiac fibroblastconditioned medium, which Zhao et al. attributed to activation of the MEK/ERK pathway [33]. We also found that fibroblast released factors were potent activators of MEK/ERK signaling, but MEK/ERK activity was not required for cytoprotection.

Taken together, we showed that factors released by human CBMSCs protect endothelial cells from the deleterious impact of nutrient and serum deprivation, but this is not a CBMSCspecific phenomenon and can be mimicked by using juvenile fibroblasts. STAT3 activation is the key mediator of cytoprotection and induces expression of the anti-apoptotic BCL-2. These results provide new insights into the complex intracellular processes that convey cytoprotection by paracrine factors and may help to better exploit the potential of cell therapy for ischemic diseases.

From the clinician's point of few, the paracrine effect of MSC-conditioned medium might be exploited by preparing cell-free products, as has, for instance, been suggested in the context of cerebral ischemia or liver regeneration [34, 35]. In the ischemic heart, such cytoprotective products may help reduce infarct size expansion after acute myocardial infarction, or possibly ameliorate the impact of "scheduled" ischemia during cardiac surgery [9].

\section{Limitations}

Deprivation of glucose and serum in an ambient atmosphere of $21 \% \mathrm{O}_{2}$ is a situation that may not reflect the actual conditions in ischemic tissue. In fact, an $\mathrm{O}_{2}$ concentration of $21 \%$ rather resembles hyperoxic conditions in situ. In consequence, we could not take reoxygenation caused injury into account, which is a major cause for cell damage after ischemia in vivo. Although our observed endpoints, survival and proliferation, are essential for neoangiogenesis, we did not directly assess endothelial cell function.

\section{Acknowledgements}

This work was supported by the Federal Ministry of Education and Research via the Reference and Translation Center for Cardiac Stem Cell Therapy at Rostock University [FKZ 0312138B]. BCRT received funding by Charité Universitätsmedizin [FKZ 1315848A]; Helmholtz-Zentrum Geesthacht [FKZ 1315848B]; and BMBF [FKZ 0315848A]. A.M.B. received a DFG stipend through the Berlin-Brandenburg School for Regenerative Therapies [GSC 203].

\section{Disclosure Statement}

None declared. 


\section{Cellular Physiology and Biochemistry}

Bader et al.: Endothelial Protection by MSC

\section{References}

1 Yang SS, Kim NR, Park KB, Do YS, Roh K, Kang KS, Kim DI: A phase I study of human cord blood-derived mesenchymal stem cell therapy in patients with peripheral arterial occlusive disease. Int J Stem Cells 2013;6:37-44.

- Gupta PK, Chullikana A, Parakh R, Desai S, Das A, Gottipamula S, Krishnamurthy S, Anthony N, Pherwani A, Majumdar AS: A double blind randomized placebo controlled phase I/II study assessing the safety and efficacy of allogeneic bone marrow derived mesenchymal stem cell in critical limb ischemia. J Transl Med 2013;11:143.

- 3 Lee HC, An SG, Lee HW, Park JS, Cha KS, Hong TJ, Park JH, Lee SY, Kim SP, Kim YD, Chung SW, Bae YC, Shin YB, Kim JI, Jung JS: Safety and effect of adipose tissue-derived stem cell implantation in patients with critical limb ischemia: a pilot study. Circ J 2012;76:1750-1760.

4 Mirotsou M, Jayawardena TM, Schmeckpeper J, Gnecchi M, Dzau VJ: Paracrine mechanisms of stem cell reparative and regenerative actions in the heart. J Mol Cell Cardiol 2011;50:280-289.

-5 Liew A, O'Brien T. Therapeutic potential for mesenchymal stem cell transplantation in critical limb ischemia. Stem Cell Res Ther 2012;3:28.

-6 Nagaya N, Fujii T, Iwase T, Ohgushi H, Itoh T, Uematsu M, Yamagishi M, Mori H, Kangawa K, Kitamura $\mathrm{S}$ : Intravenous administration of mesenchymal stem cells improves cardiac function in rats with acute myocardial infarction through angiogenesis and myogenesis. Am J Physiol Heart Circ Physiol 2004;287:H2670-2676.

7 Bhang SH, Lee S, Shin JY, Lee TJ, Kim BS: Transplantation of cord blood mesenchymal stem cells as spheroids enhances vascularization. Tissue Eng Part A 2012;18:2138-2147.

8 Kinnaird T, Stabile E, Burnett MS, Lee CW, Barr S, Fuchs S, Epstein SE: Marrow-derived stromal cells express genes encoding a broad spectrum of arteriogenic cytokines and promote in vitro and in vivo arteriogenesis through paracrine mechanisms. Circ Res 2004;94:678-685.

-9 Timmers L, Lim SK, Hoefer IE, Arslan F, Lai RC, van Oorschot AA, Goumans MJ, Strijder C, Sze SK, Choo A, Piek JJ, Doevendans PA, Pasterkamp G, de Kleijn DP: Human mesenchymal stem cell-conditioned medium improves cardiac function following myocardial infarction. Stem Cell Res 2011;6:206-214.

10 Kim SW, Han H, Chae GT, Lee SH, Bo S, Yoon JH, Lee YS, Lee KS, Park HK, Kang KS: Successful stem cell therapy using umbilical cord blood-derived multipotent stem cells for Buerger's disease and ischemic limb disease animal model. Stem Cells 2006;24:1620-1626.

11 Kern S, Eichler H, Stoeve J, Kluter H, Bieback K. Comparative analysis of mesenchymal stem cells from bone marrow, umbilical cord blood, or adipose tissue. Stem Cells 2006;24:1294-1301.

12 Bader AM, Brodarac A, Klose K, Bieback K, Choi YH, Kurtz A, Stamm C: Mechanisms of paracrine cardioprotection by cord blood mesenchymal stromal cells. Eur J Cardiothorac Surg 2014;45:983-992.

13 Klose K, Roy R, Brodarac A, Kurtz A, Ode A, Kang KS, Bieback K, Choi YH, Stamm C: Impact of heart failure on the behavior of human neonatal stem cells in vitro. J Transl Med 2013;11:236.

14 Thery C, Amigorena S, Raposo G, Clayton A: Isolation and characterization of exosomes from cell culture supernatants and biological fluids. Curr Protoc Cell Biol 2006;Chapter 3:Unit 3.22. Doi: 10.1002/0471143030.cb0322s30.

-15 Anwar S, Speciale A, Fratantonio D, Cristani M, Saija A, Virgili F, Cimino F: Cyanidin-3-O-glucoside modulates intracellular redox status and prevents HIF-1 stabilization in endothelial cells in vitro exposed to chronic hypoxia. Toxicol Lett 2014;226:206-213.

16 Yang YY, Shang J, Liu HG: Role of endoplasmic reticular stress in aortic endothelial apoptosis induced by intermittent/persistent hypoxia. Chin Med J (Engl) 2013;126:4517-4523.

17 Lee C, Cheng W, Chang M, Su Y, Chen C, Hsieh F: Hypoxia-induced apoptosis in endothelial cells and embryonic stem cells. Apoptosis 2005;10:887-894.

18 Zhang HC, Liu XB, Huang S, Bi XY, Wang HX, Xie LX, Wang YQ Cao XF, Lv J, Xiao FJ, Yang Y, Guo ZK: Microvesicles derived from human umbilical cord mesenchymal stem cells stimulated by hypoxia promote angiogenesis both in vitro and in vivo. Stem Cells Dev 2012;21:3289-3297.

- 19 Bian S, Zhang L, Duan L, Wang X, Min Y, Yu H: Extracellular vesicles derived from human bone marrow mesenchymal stem cells promote angiogenesis in a rat myocardial infarction model. J Mol Med (Berl) 2014;92:387-397. 
Bader et al.: Endothelial Protection by MSC

20 Togel F, Weiss K, Yang Y, Hu Z, Zhang P, Westenfelder C: Vasculotropic, paracrine actions of infused mesenchymal stem cells are important to the recovery from acute kidney injury. Am J Physiol Renal Physiol 2007;292:F1626-1635.

21 Zhang B, Yang S, Zhang Y, Sun Z, Xu W, Ye S: Co-culture of mesenchymal stem cells with umbilical vein endothelial cells under hypoxic condition. J Huazhong Univ Sci Technolog Med Sci 2012;32:173-180.

22 Zachary I: VEGF signalling: integration and multi-tasking in endothelial cell biology. Biochem Soc Trans 2003;31:1171-1177.

23 Steelman LS, Chappell WH, Abrams SL, Kempf RC, Long J, Laidler P, Mijatovic S, Maksimovic-Ivanic D, Stivala F, Mazzarino MC, Donia M, Fagone P, Malaponte G, Nicoletti F, Libra M, Milella M, Tafuri A, Bonati A, Basecke J, Cocco L, Evangelisti C, Martelli AM, Montalto G, Cervello M, McCubrey JA: Roles of the Raf/MEK/ ERK and PI3K/PTEN/Akt/mTOR pathways in controlling growth and sensitivity to therapy-implications for cancer and aging. Aging (Albany NY) 2011;3:192-222.

24 Hung SC, Pochampally RR, Chen SC, Hsu SC, Prockop DJ: Angiogenic effects of human multipotent stromal cell conditioned medium activate the PI3K-Akt pathway in hypoxic endothelial cells to inhibit apoptosis, increase survival, and stimulate angiogenesis. Stem Cells 2007;25:2363-2370.

25 Fujio Y, Maeda M, Mohri T, Obana M, Iwakura T, Hayama A, Yamashita T, Nakayama H, Azuma J: Glycoprotein 130 cytokine signal as a therapeutic target against cardiovascular diseases. J Pharmacol Sci 2011;117:213222.

-26 Aggarwal BB, Kunnumakkara AB, Harikumar KB, Gupta SR, Tharakan ST, Koca C, Dey S, Sung B: Signal transducer and activator of transcription-3, inflammation, and cancer: how intimate is the relationship? Ann N Y Acad Sci 2009;1171:59-76.

27 Przybyt E, Krenning G, Brinker MG, Harmsen MC: Adipose stromal cells primed with hypoxia and inflammation enhance cardiomyocyte proliferation rate in vitro through STAT3 and Erk1/2. J Transl Med 2013;11:39.

28 Iso Y, Rao KS, Poole CN, Tarikuz Zaman AK, Curril I, Sobel BE, Kajstura J, Anversa P, Spees JL: Priming with ligands secreted by human stromal progenitor cells promotes grafts of cardiac stem/progenitor cells after myocardial infarction. Stem Cells 2014;32:674-683.

29 Shabbir A, Zisa D, Lin H, Mastri M, Roloff G, Suzuki G, Lee T: Activation of host tissue trophic factors through JAK-STAT3 signaling: a mechanism of mesenchymal stem cell-mediated cardiac repair. Am J Physiol Heart Circ Physiol 2010;299:H1428-1438.

-30 Bhattacharya S, Ray RM, Johnson LR: STAT3-mediated transcription of Bcl-2, Mcl-1 and c-IAP2 prevents apoptosis in polyamine-depleted cells. Biochem J 2005;392:335-344.

-31 Choi HJ, Smithgall TE: HIV-1 Nef promotes survival of TF-1 macrophages by inducing Bcl-XL expression in an extracellular signal-regulated kinase-dependent manner. J Biol Chem 2004;279:51688-51696.

-32 Kun D, Xiang-Lin C, Ming Z, Qi L: Chlamydia inhibit host cell apoptosis by inducing Bag-1 via the MAPK/ ERK survival pathway. Apoptosis 2013;18:1083-1092.

33 Zhao L, Eghbali-Webb M: Release of pro- and anti-angiogenic factors by human cardiac fibroblasts: effects on DNA synthesis and protection under hypoxia in human endothelial cells. Biochim Biophys Acta 2001;1538:273-282.

-34 Tsai MJ, Tsai SK, Hu BR, Liou DY, Huang SL, Huang MC, Huang WC, Cheng H, Huang SS: Recovery of neurological function of ischemic stroke by application of conditioned medium of bone marrow mesenchymal stem cells derived from normal and cerebral ischemia rats. J Biomed Sci 2014;21:5.

35 Du Z, Wei C, Cheng K, Han B, Yan J, Zhang M, Peng C, Liu Y: Mesenchymal stem cell-conditioned medium reduces liver injury and enhances regeneration in reduced-size rat liver transplantation. J Surg Res 2013;183:907-915. 